\title{
Análise da qualidade de lotes de sementes de Handroanthus chrysotrichus (Mart. Ex DC.) Mattos por meio de testes de
}

\section{condutividade elétrica}

A falta de conhecimento de técnicas e dificuldade em adquirir sementes e mudas de espécies nativas florestais dificultam a implementação de experiências silviculturais apesar da sua grande importância econômica. Desse modo objetivou-se neste estudo avaliar os efeitos das combinações de fatores para análise de vigor por meio de teste de condutividade elétrica adaptando protocolos para sementes de Handroanthus chrysotrichus (Mart. Ex DC.) Mattos. No teste de condutividade elétrica utilizou-se três lotes de sementes de Handroanthus chrysotrichus com períodos de armazenamento distintos (lote I=24meses, lote II=11meses, III= três semanas), e foram testadas diferentes combinações entre volumes de água ( 40 e $80 \mathrm{~mL}$ ), quantidade de sementes ( 20 e 40 sementes), temperatura $\left(25\right.$ e $\left.35 \mathrm{C}^{\circ}\right)$, e tempo de embebição de sementes (12 e 24 horas). 0 teste de germinação foi realizado com intuito de validação do teste de vigor onde avaliou-se os parâmetros de germinação final e índice de velocidade de germinação. Todas as combinações avaliadas foram eficientes para análise dos lotes, com os lotes I e II, sempre apresentado maiores valores de condutividade, e menor porcentagem de germinação e Ivg. Assim, para o teste de condutividade elétrica em sementes de $\mathrm{H}$. chrysotrichus recomenda-se utilizar 20 sementes em um volume de $40 \mathrm{~mL}$ durante 12 horas de embebição sob temperatura de 35 Co.

Palavras-chave: Condutividade elétrica; Sementes florestais; Qualidade fisiológica.

\section{Quality analysis of seed lots of Handroanthus chrysotrichus (Mart. Ex DC.) Mattos through electrical conductivity tests}

\begin{abstract}
The lack of knowledge of techniques and difficulties in acquiring seeds and seedlings of native forest species makes it difficult to implement silvicultural experiments despite their great economic importance. The objective of this study was to evaluate the effects of combinations of factors for vigor analysis by means of electric conductivity test, adapting protocols for seeds of Handroanthus chrysotrichus (Mart. Ex DC.) Mattos. Three batches of H. chrysotrichus seeds with different storage periods (lot I = 24 months, lot II = 11 months, III = three weeks) were used in the electrical conductivity test, and different combinations were tested between water volume ( 40 and $80 \mathrm{~mL})$, seed ( 20 and 40 seeds), temperature $\left(25\right.$ and $\left.35 \mathrm{C}^{\circ}\right)$, and seed imbibition time (12 and 24 hours). The germination test was performed with the purpose of validation of the vigor test where the parameters of final germination and germination speed index were evaluated. All the evaluated combinations were efficient for the analysis of the lots, with lots I and II, always presenting higher values of conductivity, and lower percentage of germination and Ivg. Thus, for the electrical conductivity test on $\mathrm{H}$. chrysotrichus seeds, it is recommended to use 20 seeds in a volume of $40 \mathrm{~mL}$ during 12 hours of soaking under 35ㅇ.
\end{abstract}

Keywords: Electrical conductivity; Forest seed; Physiological quality

Topic: Ciências Florestais

Reviewed anonymously in the process of blind peer.
Received: 07/10/2020

Approved: 19/11/2020
Mariane Kaori Sasaya (iD

Universidade do Estado de Mato Grosso, Brasil

http://lattes.cnpq.br/0746690858035832

http://orcid.org/0000-0002-9159-4953

marianekaori@gmail.com

Roseline da Silva Melo (iD

Universidade do Estado de Mato Grosso, Brasil

http://lattes.cnpq.br/9874475151832297

http://orcid.org/0000-0003-4841-2541

melloroseline@gmail.com

Jullyanna Mendes da Silva Oliveira (i)

Universidade do Estado de Mato Grosso, Brasil

http://lattes.cnpq.br/6408417906697478

http://orcid.org/0000-0003-3445-8362

jullyannamel@gmail.com

\author{
Juliana Garlet (iD) \\ Universidade do Estado de Mato Grosso, Brasil \\ http://lattes.cnpq.br/5797330535113543 \\ http://orcid.org/0000-0002-0791-7060 \\ julianagarlet@unemat.br
}

Referencing this:

SASAYA, M. K.; MELO, R. S.; OLIVEIRA, J. M. S.; GARLET, J.. Análise da qualidade de lotes de sementes de Handroanthus chrysotrichus (Mart. Ex DC.) Mattos por meio de testes de condutividade elétrica. Revista Ibero Americana de Ciências Ambientais, v.11, n.6, p.103-111, 2020. DOI: http://doi.org/10.6008/CBPC2179-6858.2020.006.0009 


\section{INTRODUÇÃO}

Na Amazônia Meridional dentre os principais setores econômicos destaca-se o setor florestal, principalmente ligado ao beneficiamento de madeira de espécies nativas e de reflorestamento, desse modo a produção de mudas de espécies florestais nativas em viveiros tem sido uma atividade de extrema importância para a região. Um dos fatores limitantes para a implementação de novas experiências silviculturais na região, é a falta de conhecimento de técnicas e dificuldade em adquirir sementes e mudas de espécies nativas, tanto por empresários como por produtores familiares (DELARMELINA et al., 2014; SABOGAL et al., 2006).

Para garantir a produção de mudas saudáveis e de boa qualidade é fundamental o estado fisiológico das sementes a serem utilizadas, sendo necessário considerar aspectos físicos, sanitários, genéticos, fisiológicos, vigor, maturação entre outros. Pois esses aspectos interferem diretamente na capacidade de estabelecimento e no desenvolvimento da planta, onde podem variar entre e dentro dos lotes devido às diferenças qualitativas das sementes (AIMI et al., 2016). Assim torna-se crucial o estudo tecnológico das sementes para aumentar a eficiência dos processos envolvidos no beneficiamento das sementes. Além disso o estudo tecnológico das sementes também possui importância no controle de qualidade de sementes através da avaliação da germinação e do vigor de sementes, desse modo podendo auxiliar nas tomadas de decisões nas diferentes etapas do processo de produção florestal (FESSEL et al., 2010).

As espécies do gênero Handroanthus (Mattos) pertencem à família Bignoniaceae, e tem origem no Brasil, e há ocorrência de 27 espécies com distribuição geográfica ampla que inicia desde o Norte e até o sul do país incluindo a região da Amazônia Meridional. As espécies deste gênero possuem forma de vida arbórea ou arbusto, e a principal forma de propagação das espécies do gênero Handroanthus (Mattos) é via seminal e possuem interesse diversificado, frequentemente utilizadas em programas de reflorestamento. Desse modo, o controle da qualidade sanitária e fisiológicas das sementes das espécies do gênero se torna fundamental para a produção de mudas de alta qualidade. Espécies do gênero Handroanthus (Mattos) possuem valor ornamental e boa qualidade de madeira assim tornando de grande importância o estudo sobre estas espécies (FANTINEL et al., 2013; FLORA DO BRASIL, 2020; SANTOS, 2017).

Existem diversos mecanismos usados para determinar a qualidade das sementes, sendo que o teste de germinação é o mais utilizado. Segundo a Regra de Análise de Sementes (BRASIL, 2009), a qual padroniza a forma da condução deste teste, o teste de germinação é um tipo de análise laboratorial conduzido sob condições controladas que determina o potencial máximo de germinação de um lote de sementes. Podendo ser utilizado para comparar a qualidade de diferentes lotes e também estimar o valor para semeadura em campo e objetiva determinar a porcentagem do número de plântulas normais da espécie que pretende analisar.

No entanto o teste de germinação frequentemente é mais demorado e fornece índices menos sensíveis do potencial fisiológico, com isso nos últimos tempos tem surgido os testes de vigor para complementar o teste de germinação. Os testes de vigor apresentam maior eficiência em identificar 
diferenças associadas ao desempenho de lotes de sementes de forma mais rápida e confiável, bem como procura destacar lotes com maior vigor para estabelecimento desta planta em diversas condições de ambiente (MARCOS FILHO et al., 2009). Desse modo o uso de testes de vigor vem sendo utilizado pelas empresas produtoras de sementes para o programa de controle de qualidade estimando o potencial de desempenho das sementes em campo, sendo em condições favoráveis ou adversas (GRZYBOWSKI et al., 2015).

Dentre os diversos testes de vigor, destaca-se o teste de condutividade elétrica, pela sua aplicabilidade e alta capacidade de obtenção de resultados mais rápidos. Este teste busca avaliar a integridade das membranas das sementes de forma indireta através da determinação da quantidade de exsudatos lixiviados na solução de onde as sementes foram submetidas à embebição, sendo que quanto maior a degradação da semente maior será a liberação da quantidade de exsudatos. Desse modo, o teste de condutividade possui capacidade de distinguir e diferenciar o potencial de vigor para cada lote de sementes, facilitando o controle de qualidade (BARBOSA et al., 2012; SPONCHIADO, 2014).

Existem vários fatores que podem afetar os resultados do teste de condutividade elétrica como: 0 tempo de embebição, quantidade de sementes, o tamanho da semente, a temperatura do ambiente durante o tempo de embebição, e o volume de água utilizada. Este fato torna explicito a importância da padronização e adaptação de protocolos para cada espécie, incluindo as espécies pertencentes à Amazônia Meridional.

Desta forma destaca-se a carência de informações sobre testes de vigor para espécies nativas, principalmente da Amazônia Meridional, demonstrando a importância da realização de estudos com espécies florestais. Neste contexto, objetivou-se avaliar neste estudo os efeitos das combinações de fatores para análise do vigor por meio de teste de condutividade elétrica de lotes de sementes de Handroanthus chrysotrichus (Mart. Ex DC.) Mattos.

\section{MATERIAIS E MÉTODOS}

O experimento foi conduzido no Ceptam (Centro de Pesquisa e Tecnologia da Amazônia Meridional) da Universidade do Estado de Mato Grosso (UNEMAT), localizado no campus II, no município de Alta Floresta. No teste de condutividade elétrica utilizou-se três lotes de sementes de $\mathrm{H}$. chrysotrichus armazenadas em sacos de papel em laboratório por período distintos (lote I=24meses, lote $I I=11$ meses, $I I I=$ três semanas), em temperatura constante de $22^{\circ} \mathrm{C}$. Sendo que o lote I foi cedido pelo ICV (Instituto Centro de Vida), e os lotes II e III, foram coletados das árvores matrizes situadas no município de Alta Floresta/MT.

\section{Teste de vigor por condutividade elétrica}

$\mathrm{Na}$ análise de condutividade elétrica, foram testadas diferentes combinações entre volume de água (40 e $80 \mathrm{~mL}$ ), quantidade de sementes (20 e 40 sementes), temperatura ( 25 e $35 \mathrm{C}^{\circ}$ ), e tempo de embebição de sementes (12 e 24 horas) para diferentes lotes (lote I, II e III) com intuito de avaliar a melhor combinação na diferenciação dos lotes de sementes de $H$. chrysotrichus.

Primeiramente as sementes foram previamente pesadas (20 e 40 sementes) em balança eletrônica 
com precisão de 0,01g, e posteriormente, estas foram inseridas em recipientes plásticos com quantidades de água deionizada pré-estabelecidas. Em seguida estas sementes foram levadas para Biochemical Oxigen Demand chamber (BOD) regulada em uma temperatura constante com intuito de realizar o processo de embebição por um período de tempo determinado. Sendo que a mensuração da condutividade elétrica da solução de embebição foi realizada por meio de condutivímetro portátil.

$O$ resultado da leitura no condutivímetro portátil foi expresso em $\mathrm{mS} \mathrm{cm}^{-1} \mathrm{~g}^{-1}$ de semente, assim tornando necessário uma transformação na unidade de medida, desse modo realizou-se multiplicação do resultado obtido por 1000 (mil), e este foi dividido pelo peso da subamostra correspondente, resultando em $\mu \mathrm{S} \mathrm{cm} \mathrm{cm}^{-1} \mathrm{~g}^{-1}$ de semente. E posteriormente os resultados da condutividade elétrica foram comparados com a porcentagem de germinação provenientes do teste de germinação para a validação do teste. Sendo que conjuntamente com o teste de germinação também foi determinado o índice de velocidade de germinação.

Com finalidade de realizar melhor análise de diferenciação de vigor entre os três lotes, efetuou-se também análise morfométrica de plântulas germinadas através das medições de comprimento da parte aérea, raiz, comprimento total com o auxílio da régua, e a espessura do colo por meio do paquímetro digital. Os pesos individuais das plântulas foram obtidos através do uso de balança de precisão de $0,01 \mathrm{~g}$.

\section{Teste de germinação}

Para a montagem do teste de germinação, as sementes foram primeiramente desinfetadas com hipoclorito de sódio em concentração de $1 \%$ em $100 \mathrm{ml}$ de água, durante cinco minutos, e posteriormente lavadas em água corrente. Segundo recomendações da Regra Brasileira de Sementes (BRASIL, 2009), após à desinfestação, as sementes foram distribuídas com espaçamento uniforme e suficiente para minimizar a competição e contaminação entre as sementes e plântulas em desenvolvimento sobre duas folhas de papel substrato tipo Germitest com uma terceira, umedecidas previamente com água destilada na quantidade equivalente a três vezes o peso do papel seco, e posteriormente estas foram embaladas em forma de rolo e inseridas em sacos plásticos transparentes de 0,04 $\mathrm{mm}$ de espessura para evitar a perda de água por evaporação. Em seguida, os tratamentos foram levados e mantidos em câmara de germinação tipo Biochemical Oxigen Demand chamber (BOD), em temperatura constante de $25^{\circ} \mathrm{C}$, com fotoperíodo de 12 horas, utilizando lâmpadas fluorescentes. Foram realizadas contagens diárias de plântulas normais para obter os valores de porcentagem de germinação final e o índice de velocidade de germinação.

Durante todo o tempo do experimento, realizou-se o umedecimento do substrato de modo que os substratos fiquem suficientemente úmido a fim de proporcionar às sementes a quantidade de água necessária para sua germinação. Vale ressaltar que o substrato, especialmente o de papel, não deve ser tão umedecido a ponto de formar uma película de água em torno das sementes, já que este excesso restringe a aeração prejudicando a germinação (BRASIL ,2009).

A determinação da porcentagem de sementes germinadas (germinação final), realizou- se por meio da contagem do número de plântulas normais da espécie em análise, conforme a Regra Brasileira de Sementes (BRASIL, 2009). Segundo esta, as plântulas normais são aquelas que possuem todas as suas 
estruturas essenciais bem desenvolvidas, completas, proporcionais e sadias (sistema radicular, parte aérea, gemas terminais, e cotilédones), com potencial para a continuação do seu desenvolvimento e dar origem a plantas normais, quando desenvolvidas sob condições favoráveis. Em contrapartida, as plântulas anormais são aquelas que apresentam qualquer uma das suas estruturas essenciais ausentes ou tão danificadas, deformadas, e deterioradas que não mostram potencial para continuar seu desenvolvimento e dar origem a plantas normais, mesmo crescendo em condições favoráveis.

Seguindo a orientação da Regra Brasileira de Sementes (BRASIL, 2009), durante a contagem das plântulas normais, as sementes que no final do teste não germinaram, que não estavam duras, nem dormentes, onde apresentaram-se amolecidas, atacadas por micro-organismos e não apresentam nenhum sinal de início de germinação constatou-se como sementes mortas.

No presente experimento, os valores percentuais de germinação final (GF) foram obtidos por meio da Equação 1.

Equação 1:

\section{$\mathrm{GF}=\left(\sum \mathrm{n}^{\circ}\right.$ sementes germinadas $\left.* 100\right)$}

$\mathrm{n}^{\circ}$ sementes por repetição

O índice de velocidade de germinação (IVG) foi realizado conjuntamente com o teste de germinação, desse modo computou-se o número de sementes germinadas por dia, onde divide-se esse número pelo número de dias transcorridos da data de semeadura.

A seguir o índice de velocidade de germinação foi calculado por meio da Equação 2:

$$
\text { Equação 2: }
$$

$$
I V G=G 1 / N 1+G 2 / N 2+G 3 / N 3 \ldots+G n / N n
$$

Sendo: G1, G2, G3, ..., Gn = número de plântulas computadas na primeira, segunda, terceira e última contagem; N1, $\mathrm{N} 2, \mathrm{~N} 3, \ldots, \mathrm{Nn}=$ número de dias da semeadura à primeira, segunda, terceira e última contagem.

\section{Análise estatística}

Os dados obtidos neste estudo foram analisados utilizando o software Sisvar 5.6 (FERREIRA, 2014), por meio de análise de variância, e teste de Tukey $(p<0,05)$ para comparar as médias. Sendo que o teste de condutividade foi instalado em esquema fatorial analisando-se: duas quantidades de sementes, dois volumes de água, dois tempos de exposição, em duas temperaturas. Procedeu-se a análise de variância com intuito de avaliar a interação dos fatores. O teste de germinação realizado com intuito de comparação do teste de condutividade foi adotado em delineamento inteiramente casualizado (DIC), de três tratamento (lote I, lote II, e lote III) com quatro repetições, de 25 sementes cada totalizando 100 sementes para cada tratamento.

\section{RESULTADOS E DISCUSSÃO}

A seguir nas Tabelas 1 e 2, apresenta-se os valores médios de condutividade elétrica, a porcentagem de germinação final e índice de velocidade de germinação para combinações entre volumes de água (40 e 80 $\mathrm{mL}$ ), quantidade de sementes (20 e 40 sementes), temperatura ( 25 e $35 \mathrm{C}^{\circ}$ ), e tempo de embebição de sementes (12 e 24horas) para três lotes com diferentes períodos de armazenamento de sementes de $H$. 
chrysotrichus. Como não houve interação entre os fatores analisados, os dados são apresentados separadamente para os diferentes períodos de embebição avaliados.

Tabela 1: Média de condutividade elétrica $(\mu \mathrm{cm} / \mathrm{g})$, germinação e índice de germinação final para diferentes parâmetros avaliados com 12 horas de embebição de sementes de $H$. chrysotrichus.

$25 \mathrm{C}^{\circ}$

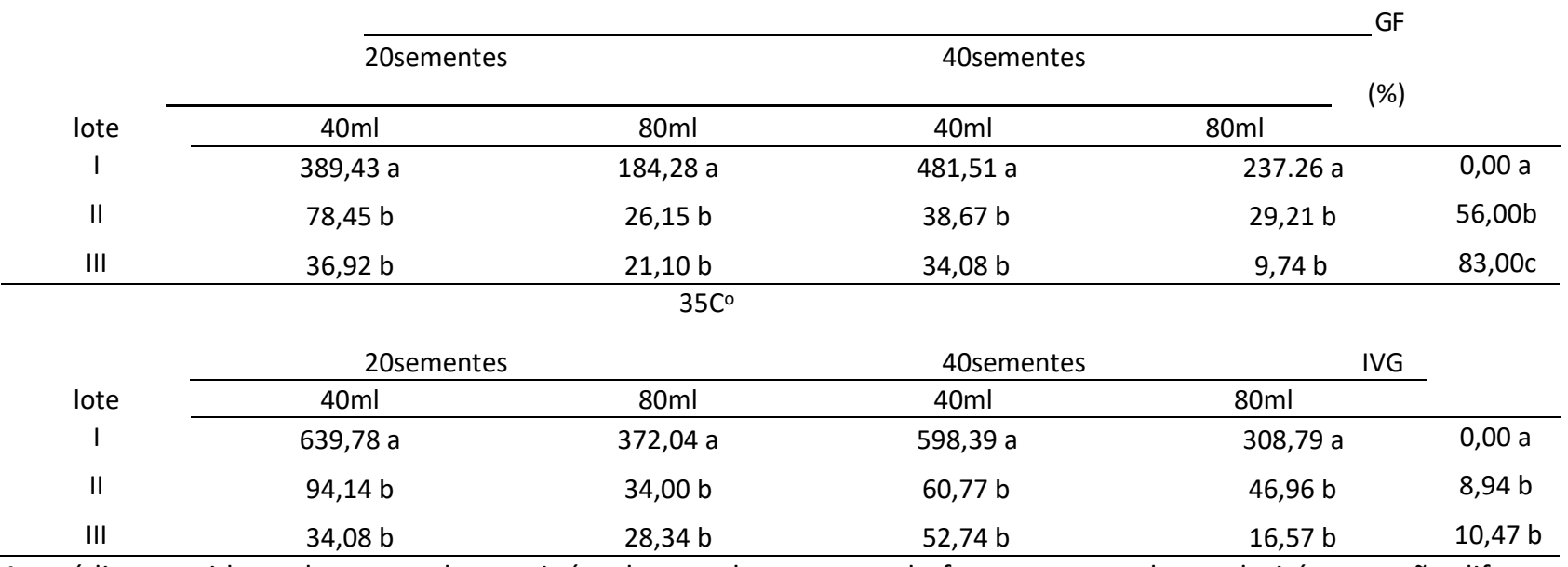

As médias seguidas pela mesma letra minúscula na coluna para cada fator e para cada condutivímetro não diferem estatisticamente entre si pelo teste de Tukey $(p<0,05)$. Lotes: I = (armazenado por 24 meses), II (armazenado por 11 meses), III (armazenado por três semanas); GF (\%):germinação final; IVG= Índice de velocidade de germinação.

Tabela 2: Média de condutividade elétrica $(\mu \mathrm{cm} / \mathrm{g})$, germinação e índice de germinação final para diferentes parâmetros avaliados com 24 horas de embebição de sementes de $H$. chrysotrichus.

$25 \mathrm{C}^{\circ}$

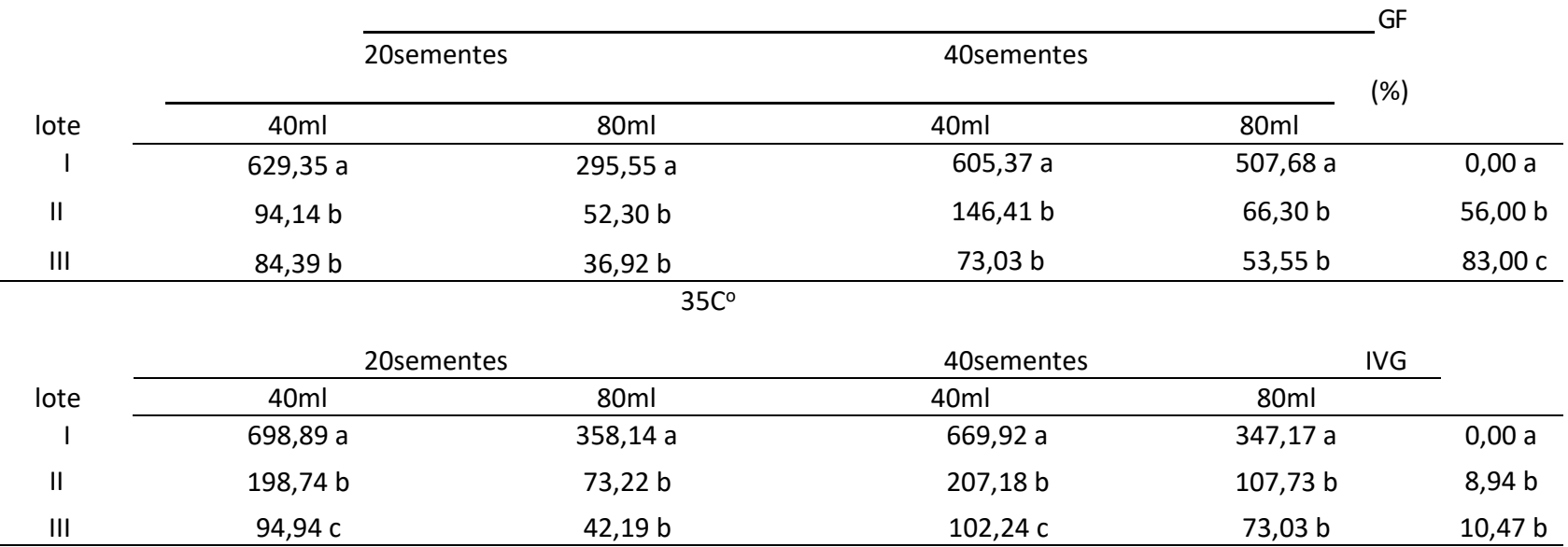

As médias seguidas pela mesma letra minúscula na coluna para cada fator e para cada condutivímetro não diferem estatisticamente entre si pelo teste de Tukey $(p<0,05)$. Lotes: I = (armazenado por 24 meses), II (armazenado por 11 meses), III (armazenado por três semanas); GF (\%):germinação final; IVG= Índice de velocidade de germinação.

De acordo com as Tabelas 1 e 2, é possível observar a diferenciação entre os lotes para todas as combinações, onde o lote III apresentou menor valor de condutividade elétrica, o que indica que este possui maior vigor germinativo, e o lote I apresentou maior valor de condutividade elétrica significando que possui menor vigor germinativo. Este fato ocorreu devido ao lote I ter maior período de armazenamento em comparação aos lotes II e III. E esse comportamento de vigor também pode ser observado através da porcentagem da Germinação final (GF) e do índice de velocidade de germinação (IVG), onde o lote III apresentou maior porcentagem de germinação e índice de velocidade de germinação, seguido pelo lote II e o lote I, como menor porcentagem de germinação, assim comprovando que o lote III, apresentava maior vigor, e o lote I menor vigor. Segundo Souza et al. (2016), períodos longos de armazenamento dos lotes de 
sementes diminuem o vigor das sementes e isso tende a aumentar o valor de condutividade elétrica desse modo contribuindo com a diferenciação de qualidade das sementes nos diferentes lotes avaliados.

Quanto à quantidade de sementes, nota-se que as combinações que utilizaram 40 sementes apresentaram maiores resultados de condutividade quando comparadas com as combinações de 20 sementes permitindo a melhor diferenciação entre os lotes. Ataide et al. (2012) também detectaram alterações significativas em combinações específicas de número de sementes, tempo de embebição e volume de água para sementes de Pterogyne nitens Tull. Com isso a utilização de quantidades maiores de sementes aumenta o valor de condutividade elétrica e permite melhor diferenciação entre lotes.

Em relação à volume de água, $40 \mathrm{ml}$ obteve-se um maior valor de condutividade elétrica quando comparado ao de $80 \mathrm{ml}$, indicando que melhores resultados são obtidos com o uso de menor volume de água, pois ao aumentar-se o volume de água de embebição das sementes há uma diminuição notável nos valores da condutividade. Esse comportamento também foi verificado por Dalanhol et al. (2014) e Ataide et al. (2012) que observaram diminuição dos valores de condutividade elétrica com o aumento do volume de água para as espécies Bowdichia virgilioides Kunth e Pterogyne nitens Tull respectivamente. Torres et al. (2015) explicam que este fato ocorre devido ao aumento de concentração dos solutos liberados pelas sementes na solução correspondente a redução do volume de água.

Quando se trata de temperatura, observa-se que para todas as combinações para os três lotes, o valor de condutividade elétrica foi maior em temperatura de $35 \mathrm{C}^{\circ}$ em relação aode $25 \mathrm{C}^{\circ}$, assim permitindo a melhor diferenciação entre os lotes. De acordo com Marques et al. (2002), este fato também foi observado em Dalbergia nigra (VELL.) Fr.All. ex Benth. onde verificaram também o aumento de valores de condutividade elétrica em temperatura mais elevadas, devido ao aumento da quantidade e a velocidade de liberação de eletrólitos lixiviados. Quando se compara os valores das Tabelas 1 e 2, é possível observar que as taxas de condutividade elétrica do período de embebição de 24 horas foram maiores que 12 horas, independente das combinações os valores de condutividade elétrica são maiores devido maior quantidade de exsudados liberados na solução de embebição. Esse resultado corroborou com o resultado de diversos autores como de Flavio et al. (2010) que realizaram o teste de condutividade para espécie Dictyoloma vandellianum A. Juss. e Dalanhol et al. (2014) que também realizaram o teste de condutividade para espécie Bowdichia virgilioides Kunth, onde maiores valores de condutividade elétrica foram observados conforme o aumento do tempo de embebição das sementes.

Desse modo, de acordo com os resultados, qualquer quantidade de semente bem como volume de água e temperatura testadas podem ser utilizadas para realizar o teste, visto que, estes não diferenciaram entre tratamentos, mas sim entre lotes. Esta diferenciação deve-se ao fato da diferença de tempo de armazenamento das sementes sendo possível recomendar para avaliação de lotes de sementes de $H$. chrysotrichus com 20 sementes em um volume de $40 \mathrm{~mL}$ durante 12 horas de embebição sob temperatura de $35 \mathrm{C}^{\circ}$. A seguir na Figura 1, são apresentas as análises morfométricas das plântulas de $H$. chrysotrichus provenientes de diferentes lotes. 


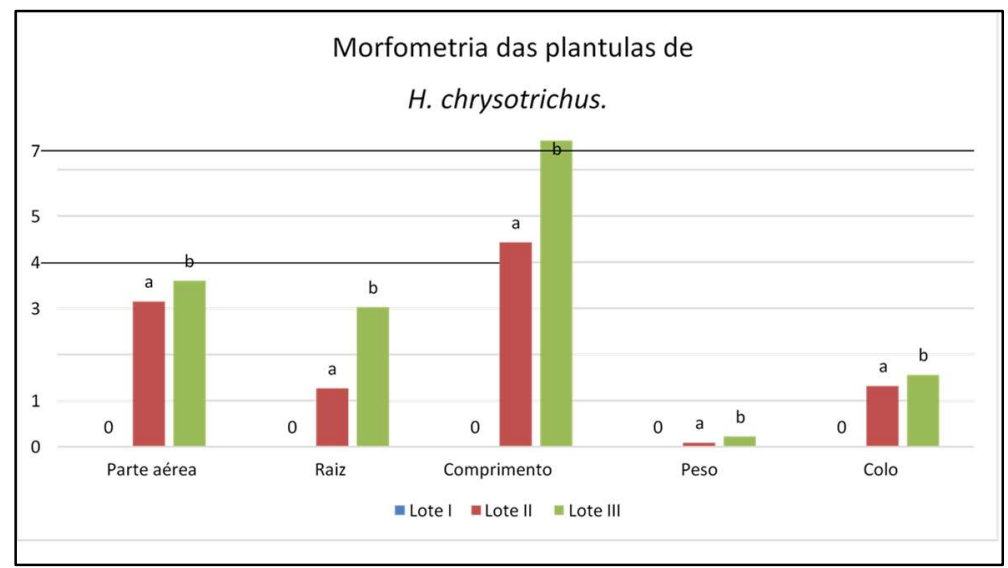

Figura 1 Gráfico da morfometria das plântulas de $H$. chrysotrichus. Médias seguidas pela mesma letra nas colunas para cada parâmetro analisado não diferem estatisticamente pelo teste de Tukey $(p<0,05)$.

Na Figura 1, é possível notar que ocorreu diferenciação entre os três lotes para todas as variáveis morfométricas analisadas, onde o lote III que teve menor período de armazenamento apresentou valores maiores, em relação ao lote II que possuía maior período de armazenamento. Para o lote I não foi possível realizar as medições devido que a porcentagem de germinação deste lote foi zero. Este resultado também coincidiu com o a pesquisa de Abbade et al. (2014), que avaliaram a viabilidade das sementes de ipê-branco (Tabebuia roseoalba (Ridl.) Sandwith), e relatam que o período de armazenamento teve forte influência sobre a porcentagem de germinação, o comprimento e o peso de matéria seca das plântulas, e foi verificado a redução dos valores destas variáveis. Este fato pode ser explicado, pois o tempo de armazenamento tende a causar uma queda progressiva da qualidade de semente, provocada pelo processo de deterioração, e consequentemente afetando na qualidade da planta a ser formada (OLIVEIRA, 2012).

\section{CONCLUSÕES}

Foi possível diferenciar o potencial de vigor das sementes de $H$. chrysotrichus nos diferentes lotes avaliados. Sendo que, quanto maior o período de armazenamento dos lotes, maior é o valor de condutividade elétrica. Assim para o teste de condutividade elétrica da espécie de $H$. chrysotrichus recomenda-se utilizar 20 sementes em um volume de $40 \mathrm{~mL}$ durante 12 horas de embebição sob temperatura de $35 \mathrm{C}^{\circ}$.

\section{REFERÊNCIAS}

ABBADE, L. C.; TAKAKI, M.. Teste de tetrazólio para avaliação da qualidade de sementes de Tabebuia roseoalba (Ridl.) Sandwith - bignoniaceae, submetidas ao armazenamento. Árvore, v.38, n.2, p.233-240, 2014.

AIMI, S. C.; ARAUJO, M. M.; MUNIZ, M. F.; WALKER, C.. Teste de sanidade e germinação em sementes de Cabralea canjerana (Vell.) Mart. Ciência Florestal, v.26, n.4, p.13611370, 2016

ATAIDE, G. M.; FLORES, A. V.; BORGES, E. L.; RESENDE, R. T.. Adequação da metodologia do teste de condutividade elétrica para sementes de Pterogyne nitens Tull. Revista Brasileira de Ciências Agrarias, v.7, n.4, p.635-640, 2012.
BARBOSA, R. M.; SILVA, C. B.; MEDEIROS, M. A.; CENTURION, M. A. P. C.; VIEIRA, R. D.. Condutividade elétrica em função do teor de água inicial de sementes de amendoim. Ciência Rural, v.42, n.1, p.45-51, 2012.

BRASIL. Ministério da Agricultura, Pecuária e Abastecimento. Regras para análise de sementes. Secretaria de Defesa Agropecuária. Brasília: Mapa, 2009.

DALANHOL, S. J.; REZENDE, E. H.; ABREU, D. C. A.; NOGUEIRA, A. C.. Teste de Condutividade Elétrica em Sementes de Bowdichia virgilioides Kunth. Floresta e Ambiente, v.21, n.1, p.69-77, 2014.

DELARMELINA, W. M.; CALDEIRA, M. V. W.; FARIA, J. C. T.; GONÇALVES, E. O; ROCHA, R. L. F.. Diferentes Substratos 
para a Produção de Mudas de Sesbania virgata. Floresta e Ambiente, v.21, n.2, p.224-233, 2014.

FANTINEL, V. S.; OLIVEIRA, L. M.; MUNIZ, M. F. B.; ROCHA, E. C.. Detecção de fungos e transmissão de Alternaria via Sementes de ipê-amarelo, Handroanthus chrysotrichus (Mart. Ex DC) Mattos. Ciências Ambientais, v.7, n.2, p.05-14, 2013.

FERREIRA, D. F.. Sisvar: a guide for its Bootstrap procedures in multiple comparisons. Science and Agrotechnology, v.38, n.2, p.109-112, 2014.

FESSEL, S. A.; PANOBIANCO, M.; SOUZA, C. R.; VIEIRA, R. D.. Teste de condutividade elétrica em sementes de soja armazenadas sob diferentes temperaturas. Bragantia, v.69, n.1, p.207-214, 2010.

FLAVIO, J. J. P.; PAULA, R. C.. Testes de envelhecimento acelerado e de condutividade elétrica em sementes de Dictyoloma vandellianum A. Juss. Scientia Forestalis, v.38, n.87, p.391-399, 2010.

FLORA DO BRASIL 2020. Handroanthus. Flora do Brasil, 2020.

GRZYBOWSKI, C. R. S.; VIEIRA, R. D.; PANOBIANCO, M. Testes de estresse na avaliação do vigor de sementes de milho. Ciência Agronômica, v.46, n.3, p.590-596, 2015.

MARCOS FILHO, J.; KIKUTI, A. L. P.; LIMA, L. B.. Métodos para avaliação do vigor de sementes de soja, incluindo a análise computadorizada de imagens. Brasileira de Sementes, v.31, n.1, p.102-112, 2009.
MARQUES, M. A.; PAULA, R. C.; RODRIGUES, T. J. D.. Adequação do teste de condutividade elétrica para determinar a qualidade fisiológica de sementes de Jacarandá-da-Bahia (Dalbergia nigra (Vell.) Fr.All. ex Benth.). Revista Brasileira de Sementes, v.24, n.1, p.271-278, 2002.

OLIVEIRA, O. S.. Tecnologia de Sementes Florestais: espécies nativas, Curitiba. UFPR, 2012.

SANTOS, S. R.. A atual classificação do antigo gênero Tabebuia (bignoniaceae), sob o ponto de vista da anatomia da madeira. Balduinia, n.58, p.10-24, 2017.

SABOGAL, C.; ALMEIDA, E.; MARMILLOD, D.; OLEGÁRIO, J. CARVALHO, P.. Silvicultura na Amazônia brasileira: avaliação de experiências e reco- mendações para implementação e melhoria dos sistemas. CIFOR, 2006.

SOUZA, G. F.; GARLET, J.; DELAZERI, P.. Teste de condutividade elétrica em sementes de Jacaranda micranta. Pesquisa Florestal Brasileira, v.36, n.85, p.79-83, 2016.

SPONCHIADO, J. C.. Teste de condutividade elétrica para determinação do potencial fisiológico de sementes de aveia branca. Ciências Agrárias, v.35, n.4, p.2405-2414, 2014.

TORRES, S. B.; PAIVA, E. P.; ALMEIDA, J. P. N.; BENEDITO, C. P.; CARVALHO, S. M. C.. Teste de condutividade elétrica na avaliação da qualidade fisiológica de sementes-de coentro. Revista Ciência Agronômica, v.46, n.3, p.622-629, 2015.

A CBPC - Companhia Brasileira de Produção Científica (CNPJ: 11.221.422/0001-03) detém os direitos materiais desta publicação. Os direitos referem-se à publicação do trabalho em qualquer parte do mundo, incluindo os direitos às renovações, expansões e disseminações da contribuição, bem como outros direitos subsidiários. Todos os trabalhos publicados eletronicamente poderão posteriormente ser publicados em coletâneas impressas sob coordenação da Sustenere Publishing, da Companhia Brasileira de Produção Científica e seus parceiros autorizados. Os (as) autores (as) preservam os direitos autorais, mas não têm permissão para a publicação da contribuição em outro meio, impresso ou digital, em português ou em tradução. 